\title{
Estimation Over Wireless Sensor Networks: Tradeoff between Communication, Computation and Estimation Qualities
}

\author{
Ling Shi ${ }^{*}$ Karl Henrik Johansson ${ }^{* *}$ Richard M. Murray ${ }^{* * *}$ \\ * Control and Dynamical Systems, California Institute of Technology, \\ Pasadena, CA 91125 USA (email: shiling@cds.caltech.edu) \\ ** School of Electrical Engineering, Royal Institute of Technology, \\ Stockholm, Sweden (email: kallej@ee.kth.se) \\ *** Control and Dynamical Systems, California Institute of Technology, \\ Pasadena, CA 91125USA (email: murray@cds.caltech.edu)
}

\begin{abstract}
:
In this paper we consider a state estimation problem over a wireless sensor network. A fusion center dynamically forms a local multi-hop tree of sensors and fuses the data into a state estimate. It is shown that the optimal estimator over a sensor tree is given by a Kalman filter of certain structure. Using estimation quality as a metric, two communication schemes are studied and compared. In scheme one, sensor nodes communicate measurement data to their parent nodes, while in scheme two, sensor nodes communicate their local state estimates to their parent nodes. We show that under perfect communication links, the two schemes produce the same estimate at the fusion center with unlimited computation at each sensor node; scheme one is always better than scheme two with limited computation. When data packet drops occur on the communication links, we show that scheme two always outperforms scheme one with unlimited computation; with limited computation, we show that there exists a critical packet arrival rate, above which, scheme one outperforms scheme two. Simulations are provided to demonstrate the two schemes under various circumstances.
\end{abstract}

\section{INTRODUCTION}

Control over large resource-constrained infrastructures requires new design paradigms beyond traditional sampleddata control. The main difficulties arise from the constrained communication and computation capabilities. Communication between network nodes is limited due to the limited energy available, particularly, if nodes are located physically far way from each other. It takes time to transfer information from one node to another, and in many cases this time increases if the information needs to be reliably delivered. Node interference, obstacles that block wireless signals, etc can sometimes cause data packets dropped in the network and hence lead to imperfect communications. Computation capability of each network node may be limited as well, for example, in a wireless sensor network, the central processing unit of each sensor node can only perform simple calculations.

We study the problem of state estimation over a wireless sensor network in this paper. The main contribution of this paper is to provide a qualitative and quantitative

\footnotetext{
* The work by L. Shi and R. M. Murray is supported in part by AFOSR grant FA9550-04-1-0169. The work by K. H. Johansson was supported by the Swedish Research Council and the Swedish Foundation for Strategic Research.
}

analysis of the tradeoff between estimation qualities and the communication and computation capabilities of each network node. In particular, we study two communication schemes and compare their performances under communication and computation constraints. We assume data are communicated over a multi-hop wireless network, instead of a single-hop network to conserve network energy usage and therefore enhance whole system life. The resulting local sensor topology has the structure of a tree for which the fusion center is the root. The quality of the state estimate depends not only on the sensor quality but also on the communication delay, i.e., the number of hops the sensor reading needs to take until it reaches the fusion center.

There are several potential application areas of the work presented in this paper, including building automation, environmental monitoring, industrial automation, power distribution, and transportation systems.

Some work related to this paper is described next. How to efficiently encode control information for event-triggered control over communication channels with severe bandwidth limitations is discussed in Bao et al. [2006].

Kalman filtering under certain information constraints, such as decentralized implementation, has been exten- 
sively studied Siljak [1978]. Implementations for which the computations are distributed among network nodes is considered in Alriksson and Rantzer [2006], Olfati-Saber [2005], Spanos et al. [2005]. Kalman filtering over lossy networks is considered in Sinopoli et al. [2004], Hespanha et al. [2007]. The interaction between Kalman filtering and how data is routed on a network seems to be less studied. Routing of data packets in networks are typically done based on the distance to the receiver node as in Bertsekas and Gallager [1991]. Some recent work addresses how to couple data routing with the sensing task using information theoretic measures was given by Liu et al. [2005].

For control over wireless sensor networks, the experienced delays and packet losses are important parameters. Randomized routing protocols that gives probabilistic guarantees on delay and loss are proposed in Bonivento et al. [2006], Lai and Paschalidis [2006].

A compensation scheme in the controller for the variations on the transport layer that such routing protocols give rise is presented in Witrant et al. [2007]. A robust control approach to control over multi-hop networks is discussed in Panousopoulou et al. [2006]. A general crosslayer approach to control and data routing seems to be an open and rather difficult topic due to many practical constraints.

The two schemes proposed in this paper have been studied for a special case (i.e., a sensor network consisting of only one node with unlimited computation capability) in Gupta et al. [2005], where the authors showed that preprocessing the measurement by the sensor before sending it to the controller is better than post-processing the measurement after receiving it by the controller when the communication link is imperfect.

The rest of the paper is organized as follows. The problem setup is described in Section 2. Some definitions and preliminary facts on Kalman filter is provided at Section 3. Optimal estimation over a sensor tree using a Kalman filter is discussed in Section 4 using both estimate and measurement communication schemes. The two schemes are further studied and compared in Section 5 under communication and computation constraints. An example is presented in Section 6 to illustrate the results obtained in previous few sections. The paper is concluded in Section 7 with a discussion on future work.

\section{PROBLEM SET-UP}

\subsection{Mathematical Models}

Consider the feedback control system in Fig. 1. The plant is given by

$$
x_{k}=A x_{k-1}+w_{k-1},
$$

where $x \in \mathbb{R}^{n}$ is the state, $w_{k}$ is white Gaussian noise with zero-mean and covariance matrix $Q \geq 0$.

A wireless sensor network is used to measure the state. The measurement equation for sensor $S_{i}$ is given by

$$
y_{k}^{i}=H_{i} x_{k}+v_{k}^{i},
$$

where $y_{k}^{i} \in \mathbb{R}^{m_{i}}$ is the measurement, $v_{k}^{i}$ is white Gaussian noise with zero-mean and covariance matrix $\Pi_{i}>0$.

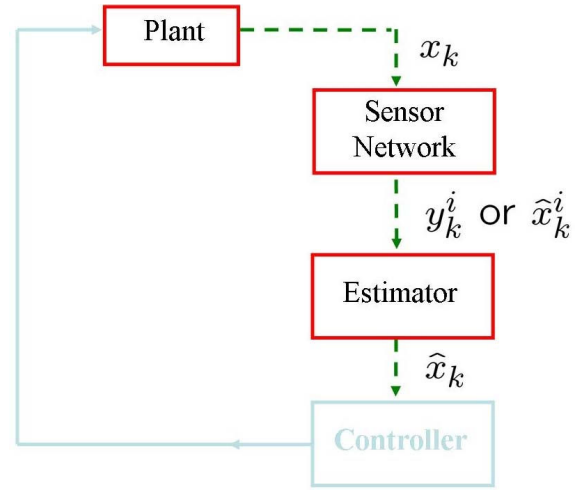

Fig. 1. Structure of Closed-Loop Control System with Measurements Gathered by a Wireless Sensor Tree

Each sensor can potentially communicate via a single-hop connection with a subset of all the sensors by adjusting its transmission power. Let us introduce a sensor $S_{0}$, which we denote the fusion center, and consider a tree $T$ with root $S_{0}$. Assume $|T|=n+1$, i.e., $T$ contains $n$ other sensor nodes. We suppose that there is a non-zero single-hop communication delay, which is smaller than the sampling time of the plant. All sensors are synchronized in time, so the data packet transmitted from $S_{i}$ to $S_{0}$ is delayed one sample when compared with the parent node of $S_{i}$.

\subsection{Problems of Interest}

We focus on the following estimation problem in the paper, thus we assume the control law is computed and sent to the plant once the controller obtains the state estimate.

Problem 2.1. Given a tree $T$ representing sensor communications with $S_{0}$, what is the optimal state estimate $\hat{x}_{k}(T)$ generated at the fusion center?

Apparently, the optimal estimation procedure depends on whether the sensors communicate raw measurement or local estimates to their parent nodes. Therefore we also discuss the two sensor communication schemes in detail and try to answer:

Problem 2.2. What is the optimal sensor communication scheme when the communication links introduce packet drops and when each sensor has limited computation power?

In the remaining sections of the paper, we provide answers to these two problems.

Remark 2.3. Although we consider link failure in this paper, i.e., by studying the effect of packet drops when sensors transmit data, we assume no sensor node failure. In case node failure does occur in practice, a new sensor communication tree can be established based on remaining set of sensors, for example, by executing the sensor reconfiguration algorithm in Shi et al. [2007].

\section{DEFINITIONS AND KALMAN FILTER PRELIMINARIES}

In this section, we provide some definitions which are used frequently in later sections. A brief introduction to Kalman Filter is also included upon which the solution to the optimal estimation problem is based. 
Define the following terms for a given a tree $T$ representing sensor communications with $S_{0}$.

- $\operatorname{Fam}_{T}\left(S_{i}\right)$ : The subtree of $T$ that is rooted at $S_{i}$.

- $\operatorname{Par}_{T}\left(S_{i}\right)$ : The parent node of $S_{i}$ in $T$.

The depth of $T$ is denoted $h_{T}$. For all notations, we drop the subscript $T$ when the considered tree follows from the context.

Next, we formalize estimation over a sensor tree under communication energy constraints, in which the operations above can be used to improve the performance.

Let us define the following state estimates and other quantities at $S_{0}$ :

$$
\begin{aligned}
\hat{x}_{k}^{-}(T) & \triangleq \mathbb{E}\left[x_{k} \mid \text { all measurements up to } k-1\right], \\
\hat{x}_{k}(T) & \triangleq \mathbb{E}\left[x_{k} \mid \text { all measurements up to } k\right], \\
P_{k}^{-}(T) & \triangleq \mathbb{E}\left[\left(x_{k}-\hat{x}_{k}^{-}(T)\right)\left(x_{k}-\hat{x}_{k}^{-}(T)\right)^{\prime}\right], \\
P_{k}(T) & \triangleq \mathbb{E}\left[\left(x_{k}-\hat{x}_{k}(T)\right)\left(x_{k}-\hat{x}_{k}(T)\right)^{\prime}\right], \\
P_{\infty}^{-}(T) & \triangleq \lim _{k \rightarrow \infty} P_{k}^{-}(T), \text { if the limit exists, } \\
P_{\infty}(T) & \triangleq \lim _{k \rightarrow \infty} P_{k}(T), \text { if the limit exists. }
\end{aligned}
$$

Consider the following discrete time system

$$
\begin{aligned}
& x_{k}=A x_{k-1}+w_{k-1} \\
& y_{k}=C_{k} x_{k}+v_{k}
\end{aligned}
$$

where $w_{k-1}$ and $v_{k}$ are white Gaussian noises with zeromean and covariances $Q \geq 0$ and $R_{k}>0$, respectively. The estimates $\hat{x}_{k}$ and $P_{k}$ can be computed as

$$
\left(\hat{x}_{k}, P_{k}\right)=\mathbf{K F}\left(\hat{x}_{k-1}, P_{k-1}, y_{k}, C_{k}, Q, R_{k}\right),
$$

where $\mathbf{K F}$ denotes the Kalman filter given by the following update equations:

$$
\begin{aligned}
\hat{x}_{k}^{-} & =A \hat{x}_{k-1}, \\
P_{k}^{-} & =A P_{k-1} A^{\prime}+Q, \\
K_{k} & =P_{k}^{-} C_{k}^{\prime}\left[C_{k} P_{k}^{-} C_{k}^{\prime}+R_{k}\right]^{-1}, \\
\hat{x}_{k} & =A \hat{x}_{k-1}+K_{k}\left(y_{k}-C_{k} A \hat{x}_{k-1}\right), \\
P_{k} & =\left(I-K_{k} C_{k}\right) P_{k}^{-} .
\end{aligned}
$$

\section{OPTIMAL ESTIMATION OVER SENSOR TREES}

In this section, we consider two sensor communication schemes. Optimal estimation over a sensor tree using each schemes is given. Then the two schemes are compared in cases when communication links introduce packet drops and when sensor nodes have limited computation power. We provide conditions on which scheme is better than the other in these cases.

\subsection{Sensor Communication Schemes}

Scheme One - Measurement Communication In this scheme, the sensors only send their raw measurement data to its parent without processing which has been used in Shi et al. [2007] to construct minimum energy sensor tree. The fusion center $S_{0} \in T$ collects data from all sensors in $T$. The role of the fusion center is to compute an estimate of $x$ and forward it to a controller node.

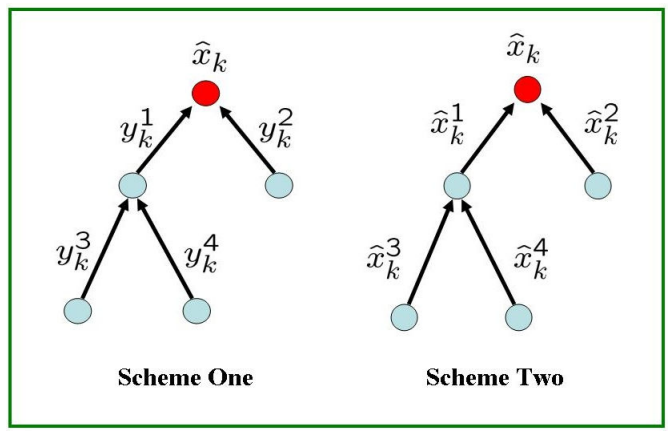

Fig. 2. Sensor Communication Schemes

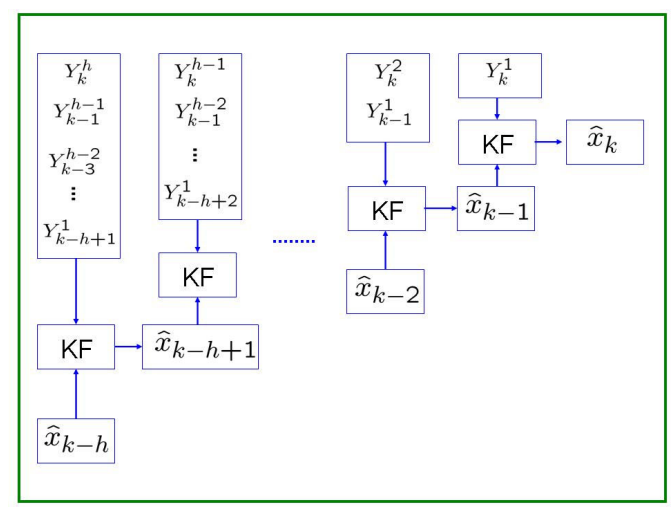

Fig. 3. Kalman Filter Iterations at Time $k$

Scheme Two - Estimate Communication In this scheme each sensor $S_{i}$ runs a local Kalman filter and computes their own state estimate $\hat{x}_{k}^{i}$ at time $k$. After $\hat{x}_{k}^{i}$ is obtained, $S_{i}$ sends $\hat{x}_{k}^{i}$ to $\operatorname{Par}\left(S_{i}\right) . \operatorname{Par}\left(S_{i}\right)$ then updates its state estimate by fusing the estimates from its children with its own. Since the computation is distributed in this scheme and each $S_{i}$ maintains a local estimate of the state, this scheme is more robust than scheme one when there is data packet drops as shown in Theorem 5.1. In principle, after fusing the estimates from its child nodes, the parent node can also send the estimate back to its child nodes and hence improve their estimation quality. The analysis in this paper extends straightforward to this case.

\subsection{Optimal Estimation Over Sensor Trees: Scheme One}

Let the tree $T$ with depth $h$ that represents the sensor communications with the FC be given. Define $\mathcal{Y}_{k}^{k-i+1}$ as all measurements available at the FC for time $k-i+1$ at time $k, i=1, \cdots, h$. Figure 3 shows the overall estimation scheme at time $k$, where

$$
Y_{k}^{l} \triangleq\left\{y_{k-l+1}^{i}: S_{i} \text { is } l \text { hop away from } S_{0}\right\}, l=1, \cdots, h .
$$

Let $S_{i_{j}}$ be the node that is $j$ hops away from $S_{0}$. Define

$$
\begin{aligned}
& \Gamma_{j} \triangleq\left[H_{1_{j}} ; H_{2_{j}} ; \cdots\right], j=1, \cdots, h \\
& C_{i} \triangleq\left[\Gamma_{1} ; \cdots ; \Gamma_{i}\right], i=1, \cdots, h \\
& \Upsilon_{j} \triangleq \operatorname{diag}\left\{\Pi_{1_{j}}, \Pi_{2_{j}}, \cdots\right\}, j=1, \cdots, h \\
& R_{i} \triangleq \operatorname{diag}\left\{\Upsilon_{1}, \cdots, \Upsilon_{i}\right\}, i=1, \cdots, h
\end{aligned}
$$

For $X \geq 0$, define

$$
g_{C_{i}}(X) \triangleq A X A^{\prime}+Q-A X C_{i}^{\prime}\left[C_{i} X C_{i}^{\prime}+R_{i}\right]^{-1} C_{i} X A^{\prime} .
$$


Theorem 4.1. Consider a sensor tree $T$ with depth $h$.

(1) $\hat{x}_{k}$ and $P_{k}$ can be computed from $h$ parallel filter as

$$
\begin{aligned}
& \left(\hat{x}_{k-h+1}, P_{k-h+1}\right) \\
= & \mathbf{K F}\left(\hat{x}_{k-h}, P_{k-h}, \mathcal{Y}_{k}^{k-h+1}, C_{h}, Q, R_{h}\right) \\
& \vdots \\
& \left(\hat{x}_{k-1}, P_{k-1}\right) \\
= & \mathbf{K F}\left(\hat{x}_{k-2}, P_{k-2}, \mathcal{Y}_{k}^{k-1}, C_{2}, Q, R_{2}\right) \\
& \left(\hat{x}_{k}, P_{k}\right) \\
= & \mathbf{K F}\left(\hat{x}_{k-1}, P_{k-1}, \mathcal{Y}_{k}^{k}, C_{1}, Q, R_{1}\right)
\end{aligned}
$$

(2) Furthermore $P_{k}^{-}$and $P_{\infty}^{-}$satisfies

$$
\begin{aligned}
& P_{k}^{-}=g_{C_{2}} \cdots \circ g_{C_{h}}\left(P_{k-h}^{-}\right) \\
& P_{\infty}^{-}=g_{C_{2}} \cdots \circ g_{C_{h-1}}\left(\bar{P}_{\infty}^{-}\right)
\end{aligned}
$$

where $\bar{P}_{\infty}$ is the unique solution to $g_{C_{h}}\left(\bar{P}_{\infty}^{-}\right)=\bar{P}_{\infty}^{-}$.

Proof: See Shi et al. [2007].

\subsection{Optimal Estimation Over Sensor Trees: Scheme Two}

In this section, we assume each sensor node has enough computation power to run a local Kalman filter and hence generate its own estimate of the state. It then communicates the estimate to its parent node.

Let $\tilde{x}_{k}^{i}$ and $\hat{x}_{k}^{i}$ be the local state estimate and $\tilde{P}_{k}^{i}$ and $P_{k}^{i}$ be the local error covariance of $S_{i}$ before and after fusing with its children's estimates respectively.

After $y_{k}^{i}$ is obtained, $S_{i}$ runs the Kalman filter in its information form as follows

$$
\begin{aligned}
\hat{x}_{k}^{i-} & =A \hat{x}_{k-1}^{i}, \\
P_{k}^{i-} & =A P_{k-1}^{i} A^{\prime}+Q \\
\left(\tilde{P}_{k}^{i}\right)^{-1} & =\left(P_{k}^{i-}\right)^{-1}+H_{i}^{\prime} \Pi_{i}^{-1} H_{i} \\
\tilde{x}_{k}^{i} & =\hat{x}_{k}^{i-}+K_{k}^{i}\left[y_{k}^{i}-H_{i} \hat{x}_{k}^{i-}\right]
\end{aligned}
$$

where $K_{k}^{i}=\tilde{P}_{k}^{i} H_{i}^{\prime} \Pi_{i}^{-1}$.

Then $S_{i}$ sends $\hat{x}_{k}^{i-}, P_{k}^{i-}, \tilde{x}_{k}^{i}, \tilde{P}_{k}^{i}$ to its parent node. Notice that for the leaf nodes, $\tilde{x}_{k}^{i}$ and $\tilde{P}_{k}^{i}$ are the same as $\hat{x}_{k}^{i}$ and $P_{k}^{i}$. For intermediate nodes, after they receive the local estimates from their children, they update the state estimate and error covariance according to

$$
\begin{aligned}
&\left(P_{k}^{i}\right)^{-1}=\left(\tilde{P}_{k}^{i}\right)^{-1}+\sum_{j: \operatorname{Par}\left(S_{j}\right)=S_{i}}\left[\left(\tilde{P}_{k}^{j}\right)^{-1}-\left(P_{k}^{j-}\right)^{-1}\right] \\
& \hat{x}_{k}^{i}=P_{k}^{i}\left\{\left(\tilde{P}_{k}^{i}\right)^{-1} \tilde{x}_{k}^{i}+\sum_{j: \operatorname{Par}\left(S_{j}\right)=S_{i}}\right. \\
&\left.\quad\left[\left(\tilde{P}_{k}^{j}\right)^{-1} \tilde{x}_{k}^{j}-\left(P_{k}^{j-}\right)^{-1} \hat{x}_{k}^{j-}\right]\right\}
\end{aligned}
$$

Denote $\hat{x}_{k}(1)$ and $\hat{x}_{k}(2)$ as the state estimate at $S_{0}$ using scheme one and two respectively. $P_{\infty}^{i-}(1)$ and $P_{\infty}^{i-}(2)$ etc, are denoted in the same way. Since $S_{i}$ is the root of subtree $\operatorname{Fam}\left(S_{i}\right)$, if we define $\tilde{\Gamma}_{j}, \tilde{C}_{i}, \tilde{\Upsilon}_{j}, \tilde{R}_{i}, g_{\tilde{C}_{i}}$ similarly as in the previous section for root $S_{0}$, we immediately obtain the following theorem.

Theorem 4.2. (1) If $\hat{x}_{0}(1)=\hat{x}_{0}(2)$ and $P_{0}(1)=P_{0}(2)$, then $\hat{x}_{k}(1)=\hat{x}_{k}(2)$ for all $k$.

$$
P_{\infty}^{i-}=g_{\tilde{C}_{2}} \cdots \circ g_{\tilde{C}_{h_{i}-1}}\left(\bar{P}_{\infty}^{i-}\right)
$$

where $h_{i}$ is the depth of $\operatorname{Fam}\left(S_{i}\right)$ and $\bar{P}_{\infty}^{i-}$ is the unique solution to

$$
g_{C_{h_{i}}}\left(\bar{P}_{\infty}^{i-}\right)=\bar{P}_{\infty}^{i-} .
$$

\section{Proof:}

(1) It is shown in Rao and Durrant-Whyte [1991] that $\hat{x}_{k}^{i}$ from Eqn (15) is the same as computed from a centralized Kalman filter where the measurements from its children are collected at $S_{i}$. Consequently, $\hat{x}_{k}$ at $S_{0}$ using scheme two is the same as using scheme one.

(2) Direct result from Theorem 4.1.

\section{COMPARISON OF THE TWO SCHEMES}

We assume when the computation power of each sensor node is limited, the local state estimate transmitted from a sensor node to its parent node is delayed by one time step using scheme two. Since using scheme one, the sensor nodes simply forward the measurement data, hence there is no delay in transmitting its data. With this assumption, the two schemes are compared in the following four scenarios when communication links introduce packet drops and when sensor nodes have limited computation power. We provide conditions on one scheme is better than the other.

\subsection{Perfect Communication with Unlimited Computation}

With no packet drops and unlimited computation, $S_{0}$ has the same estimate using either scheme as we have stated in the previous section.

\subsection{Imperfect Communication with Unlimited Computation}

With unlimited computation at each sensor node, if packet drops occur due to interference, network congestion etc, scheme two outperforms scheme one in the expected sense as shown in Theorem 5.1. Furthermore, there exist cases that scheme one is arbitrarily worse than scheme two as shown in Theorem 5.2.

Let $\gamma_{k}^{i}(1)$ and $\gamma_{k}^{i}(2)$ be the indicator functions of whether the packet from $S_{i}$ is successfully transmitted to $\operatorname{Par}\left(S_{i}\right)$ $(=1)$ or dropped $(=0)$. To compare the performance of the two schemes, assume $\gamma_{k}^{i}=\gamma_{k}^{i}(1)=\gamma_{k}^{i}(2)$ for all $k$ and $i$. Then we have the following result.

Theorem 5.1. Assume unlimited computation at each sensor node and $P_{0}^{-}(1)=P_{0}^{-}(2)$. Then

$$
\mathbb{E}\left[P_{k}^{-}(1)\right] \geq \mathbb{E}\left[P_{k}^{-}(2)\right]
$$

for all $k \geq 0$.

Proof: We give a proof for the case where there is only one sensor (see Fig. 4). The idea is straightforward to present in the general case. Since we only consider one 


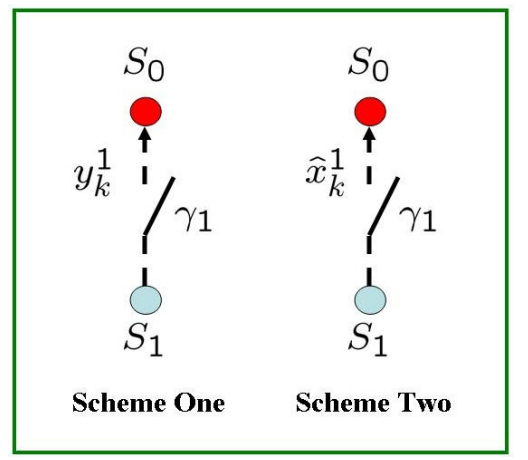

Fig. 4. Comparison of Two Communication Schemes

sensor here, we simply write $\gamma_{k}^{1}$ as $\gamma_{k}$. Then by definition of the expected value, we only need to show that

$$
P_{k}^{-}(1) \geq P_{k}^{-}(2)
$$

for a particular realization of the packet drop sequences $\gamma_{k}$. We use mathematical induction to prove Eqn (18).

(1) $P_{0}^{-}(1) \geq P_{0}^{-}(2)$ holds by assumption in the theorem.

(2) Assume $P_{m}^{-}(1) \geq P_{m}^{-}(2)$ for $0 \leq m<k$.

(3) At $m+1$.

(a) If $\gamma_{m+1}=1, P_{m+1}^{-}(2):=g^{m+1}\left(P_{0}^{-}(2)\right)$

$$
\begin{aligned}
P_{m+1}^{-}(1) & =g\left(P_{m}^{-}(1)\right) \\
& \geq g^{m+1}\left(P_{0}^{-}(1)\right) \\
& \geq g^{m+1}\left(P_{0}^{-}(2)\right) \\
& =P_{m+1}^{-}(2) .
\end{aligned}
$$

(b) If $\gamma_{m+1}=0$,

$$
\begin{aligned}
P_{m+1}^{-}(1) & =h\left(P_{m}^{-}(1)\right) \\
& \geq h\left(P_{m}^{-}(2)\right) \\
& =P_{m+1}^{-}(2) .
\end{aligned}
$$

where the inequalities are from properties of $h$ and $g$ functions and can be found in Sinopoli et al. [2004]. The three steps above complete the induction.

Theorem 5.2. Consider the one sensor case (Fig. 4). Assume unlimited computation at each sensor node and $P_{0}^{-}(1)=P_{0}^{-}(2)$. Further assume that $A$ is unstable, $\left(H_{1}, A\right)$ is detectable and $(A, \sqrt{Q})$ is controllable. Then the following are true.

(1) For any $k \geq 1$ and any packet drop sequences $\gamma_{l}, l=$ $1, \cdots, k$,

$$
P_{k}^{-}(1) \geq P_{k}^{-}(2) \text {. }
$$

(2) If $H_{1}^{-1}$ does not exist, then there exist $c_{0}>0, c>1$, a positive integer $k_{\min }$ and a packet drop sequence $\gamma_{l}, l=1, \cdots, k$, such that

$$
P_{k}^{-}(1) \geq c_{0} c^{k} P_{k}^{-}(2)
$$

for all $k \geq k_{\min }$.

(3) If $H_{1}^{-1}$ exists, then for any packet drop sequence, there does not exist $c>1$ such that Equ (20) holds.

\section{Proof:}

(1) See proof to Theorem 5.1.

(2) Assume $H_{1}^{-1}$ does not exist. We explicitly construct a class of packet drop sequences for which $c>1$ and
Eqn (20) holds. Let $\theta$ be a given positive integer. Consider the packet drop sequence

$$
\cdots 1 \underbrace{00 \cdots 00}_{\theta \text { zeros }} 1 \underbrace{00 \cdots 00}_{\theta \text { zeros }} 1
$$

i.e., $\gamma_{l}=1$ for $l \bmod (\theta+1)=1$ and $\gamma_{l}=0$ otherwise. Let $k_{\min }$ be such that

$$
g^{k_{\min }}\left(P_{0}^{-}(2)\right) \leq(1+\epsilon) P_{\infty}^{-}(2)
$$

where $0<\epsilon<1$. Such $k_{\min }$ exists as

Therefore

$$
\lim _{k \rightarrow \infty} g^{k}\left(P_{0}^{-}(2)\right)=P_{\infty}^{-}(2) .
$$

$$
P_{k}^{-}(2) \leq h^{\theta}\left((1+\epsilon) P_{\infty}^{-}(2)\right)
$$

for all $k \geq k_{\min }$. Write $k=\theta \tilde{k}+k^{\prime}$ for $k \geq k_{\min }$, where $1 \leq k^{\prime}<\theta$. Then

$$
P_{k}^{-}(1)=h^{k^{\prime}} \circ g\left(\left(h^{\theta} g\right)^{\tilde{k}}\left(P_{0}^{-}(1)\right)\right) .
$$

Since $H_{1}^{-1}$ does not exist and $A$ is unstable, we can pick a $\theta>1$ such that $\left(h^{\theta} g\right)^{\tilde{k}}$ diverges. For this $\theta$, there exists $\tilde{c}>1$ such that

$$
P_{k}^{-}(1) \geq \tilde{c}^{\tilde{k}} P_{0}^{-}(1) .
$$

Let

then

$$
c=(\tilde{c})^{\frac{1}{\theta}}, c_{0}=\frac{P_{0}^{-}(1)}{\tilde{c} h^{\theta}\left((1+\epsilon) P_{\infty}^{-}(2)\right)}
$$

$$
P_{k}^{-1}(1) \geq c_{0} c^{k} P_{k}^{-}(2) .
$$

(3) If $H_{1}^{-1}$ exists, then $g(X) \leq \bar{M}$ where $\bar{M}=$ $A H_{1}^{-1} \Pi_{1}\left(H_{1}^{\prime}\right)^{-1} A^{\prime}+Q$ according to Shi et al. [2005]. Hence there exists $k_{\min }>0$ such that if $k \geq k_{\min }$ and $\gamma_{k}=1$, we have $P_{k}^{-}(1) \leq c_{1} P_{k}^{-}(2)$ where

$$
\begin{aligned}
& \text { If } \gamma_{k}=0 \text { and let } \\
& \qquad k^{\prime}=\min _{s}\left\{s \leq k: \gamma_{s}(1)=\gamma_{s}(2)=1,\right.
\end{aligned}
$$$$
c_{1}=\frac{\bar{M}}{(1-\epsilon) P_{\infty}^{-}(2)} .
$$

then

$$
\begin{aligned}
P_{k}^{-}(1) & =h^{k-k^{\prime}}\left(P_{k^{\prime}}^{-}(1)\right) \\
& \leq h^{k-k^{\prime}}\left(c_{1} P_{k^{\prime}}^{-}(2)\right) \\
& =c_{1} h^{k-k^{\prime}}\left(P_{k^{\prime}}^{-}(2)\right) \\
& =c_{1} P_{k}^{-}(2) .
\end{aligned}
$$

Remark 5.3. Theorem 5.2 only considers the one sensor case. Generalization to multiple sensor case is straightforward. Theorems 5.1 and 5.2 show that scheme one is at most as good as and could be arbitrarily worse than scheme two depending on the packet drops. The results suggest that in a network where busty packet drops can occur, it is always better to process the measurement before sending it to its parent node assuming unlimited computation power at each sensor node. However, this does not always hold when the computation power at each sensor node is limited as shown in the next two sections.

\subsection{Perfect Communications with Limited Computation}

With limited computation at each sensor node, if there are no packet drops, scheme one is always better than scheme 
two. This is quite different from the unlimited computation case where scheme two is always better than scheme one.

Theorem 5.4. Assume limited computation at each sensor node and no packet drops occur. Then if $P_{0}^{-}(1)=P_{0}^{-}(2)$,

$$
P_{k}^{-}(1) \leq P_{k}^{-}(2) \forall k \text {. }
$$

Proof: We give a proof for the case where there is only one sensor (Fig. 4). We have assumed that transmitting the estimate to its parent node has one time delay for a sensor when using scheme two, and has no time delay when using scheme one, therefore in this case,

$$
P_{k}^{-}(1)=g\left(P_{k-1}^{-}(1)\right), P_{k}^{-}(2)=h\left(g\left(P_{k-2}^{-}(2)\right)\right) .
$$

Assume $P_{k}^{-}(1) \leq P_{k}^{-}(2)$ for all $k \leq m-1$. Then we have

$$
\begin{aligned}
P_{m}^{-}(1) & =g\left(g\left(P_{m-2}^{-}(1)\right)\right) \\
& \leq g\left(g\left(P_{m-2}^{-}(2)\right)\right. \\
& \leq h\left(g\left(P_{m-2}^{-}(2)\right)\right) \\
& =P_{m}^{-}(2)
\end{aligned}
$$

This completes the induction step as $P_{0}^{-}(1)=P_{0}^{-}(2)$.

\subsection{Imperfect Communications with Limited Computation}

With limited computation at each sensor node, if the communication network introduces packet drops, scheme one is not always better than scheme two. As we show next, there exists some critical packet arrival rate, only above which, scheme one is better. Fig. 8 in Section 6 demonstrates this phenomenon. The results is stated in the following theorem.

Theorem 5.5. Assume limited computation at each sensor node and $P_{0}^{-}(1)=P_{0}^{-}(2)$. Further assume $\left[H_{1} ; H_{2} ; \cdots ; H_{n}\right]$ does not exist. Let $\gamma_{i}(1)=\gamma_{i}(2)=\gamma_{i}$ for all $i$ and denote

$$
\gamma_{-i}=\left(\gamma_{1}, \cdots, \gamma_{i-1}, \gamma_{i+1}, \cdots, \gamma_{n}\right) \text {. }
$$

Then for fixed $\gamma_{-i}$, there exists $\bar{\gamma}_{i}$ such that if $\gamma_{i} \leq \bar{\gamma}_{i}$,

$$
\mathbb{E}\left[P_{k}^{-}(1)\right] \geq \mathbb{E}\left[P_{k}^{-}(2)\right] \forall k
$$

and otherwise if $\gamma_{i}>\bar{\gamma}_{i}$.

Proof: We give a proof for the case where there is only one sensor (Fig. 4). Again, it is straightforward to extend it in the general case. Following the same line as in the proof of Theorem 5.2 (i.e., by constructing explicit packet arrival sequences), we can show that there exists $\gamma_{i}$ such that if $\gamma_{i} \leq \underline{\gamma_{i}}$,

$$
\mathbb{E}\left[P_{k}^{-}(1)\right] \geq \mathbb{E}\left[P_{k}^{-}(2)\right]
$$

We can make the bound tighter by noticing that when $\gamma_{i}=1$ for all $i$, from Theorem 5.4,

$$
\mathbb{E}\left[P_{k}^{-}(1)\right]=P_{k}^{-}(1) \leq P_{k}^{-}(2)=\mathbb{E}\left[P_{k}^{-}(2)\right]
$$

Since $\mathbb{E}\left[P_{k}^{-}(1)\right]$ and $\mathbb{E}\left[P_{k}^{-}(2)\right]$ are continuous and decreasing functions with respect to $\gamma_{i}$, from real analysis, we know there exists $\underline{\gamma_{i}} \leq \bar{\gamma}_{i} \leq 1$ such that if $\gamma_{i} \leq \bar{\gamma}_{i}$,

$$
\mathbb{E}\left[P_{k}^{-}(1)\right] \geq \mathbb{E}\left[P_{k}^{-}(2)\right] \forall k
$$

and otherwise if $\gamma_{i}>\bar{\gamma}_{i}$.

\section{EXAMPLES}

We consider an integrator chain as an example in this section to demonstrate the performance of the two schemes

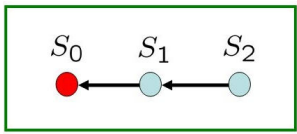

Fig. 5. Integrator Chain Example
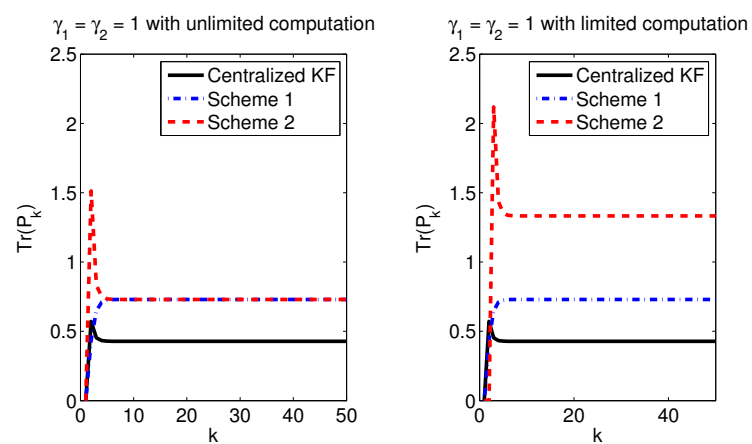

Fig. 6. Comparison of Two Schemes Under Perfect Communication

under various circumstances. The discrete time system dynamics is given by Eqn (1) with

$$
A=\left[\begin{array}{cc}
1 & 0.1 \\
0 & 1
\end{array}\right] \text {. }
$$

with process noise covariance $Q=0.3 I$. There are two sensors available. The measurement equations are given by

$$
\begin{aligned}
& y_{k}^{1}=\left[\begin{array}{ll}
0 & 1
\end{array}\right] x_{k}+v_{k}^{1}, \\
& y_{k}^{2}=\left[\begin{array}{ll}
1 & 0
\end{array}\right] x_{k}+v_{k}^{2}
\end{aligned}
$$

where $v_{k}^{i}$ are white Gaussian with zero-mean and covariances $\Pi_{1}=0.25$ and $\Pi_{2}=0.5$. Assume sensor $i$ is $i$ hops away from $S_{0}$ (Fig. 5).

Notice that $A$ is unstable in this example. In Fig. 6, we plot the error covariance evolution of the two schemes. We also plot the centralized Kalman filter solution. The figure on the left hand side show that the two schemes produce the same error covariance and it is bigger than the solution from the centralized Kalman filter, which is as expected. The figure on the right side shows the case with limited computation at the sensor nodes. It shows in this case that scheme two produces a worse result than scheme one, which is stated in Theorem 5.4.

In Fig. 7, we plot the difference of the error covariance for the two schemes. The top figure shows that scheme one is always worse than scheme two after the transient period with imperfect communication and unlimited computation at each sensor node, which is captured in Theorem 5.2. The bottom figure shows that when the packet drop rates are low, the expected value of the error covariance from scheme one is smaller than that from scheme two, which corresponds to Theorem 5.5.

In Fig. 8, we fix $\gamma_{2}$ at different values and plot the average of $\operatorname{Trace}\left(P_{k}(1)\right)$ and $\operatorname{Trace}\left(P_{k}(1)\right)$ respectively. It shows that when $\gamma_{2}$ is relatively big, i.e., $\gamma_{2}=0.8$, scheme one starts to become worse than scheme two after $\gamma_{1}$ becomes smaller than 0.3 ; when $\gamma_{2}$ is relatively small, i.e., $\gamma_{2}=0.4$, scheme one starts to become worse than scheme two after 

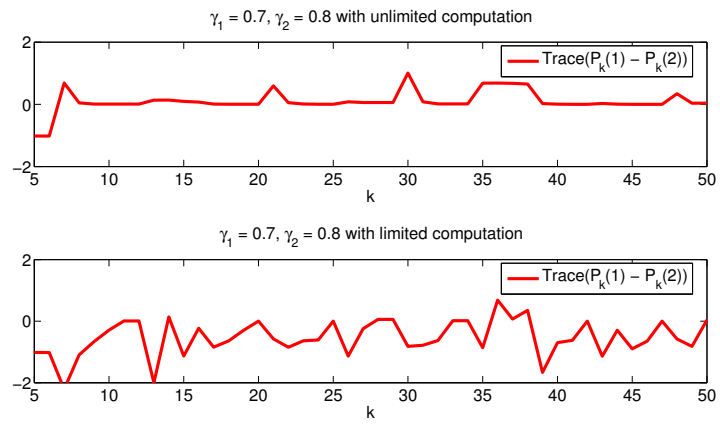

Fig. 7. Comparison of Two Schemes Under Imperfect Communication
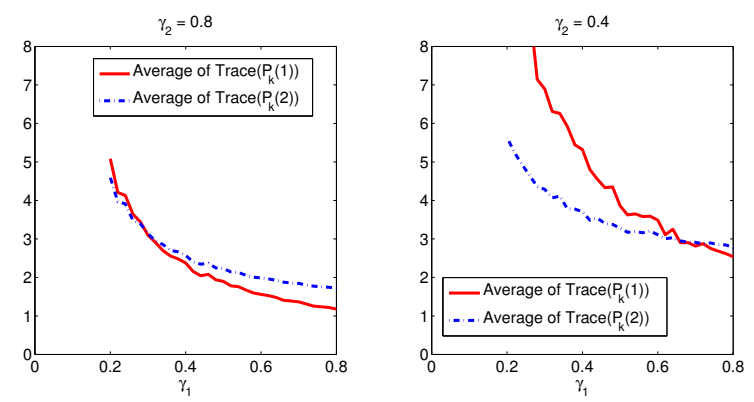

Fig. 8. Comparison of Two Schemes With Limited Computation

$\gamma_{1}$ becomes smaller than 0.63 . The results agree with Theorem 5.5.

\section{CONCLUSION AND FUTURE WORK}

In this paper, we consider an estimation problem over wireless sensor networks. We derive optimal estimation for a sensor tree and study two communication schemes. We show that the two schemes have distinct performance with/without stochastic packet drops and with/without unlimited computation at each sensor node. We also provide conditions on which scheme is better in different scenarios.

There are a few extensions of this work that will be pursued in the near future, which includes: closing the loop over the network using the estimation schemes we have proposed; finding the closed form solution to the critical packet arrival rate in Theorem 5.5; conducting experiments using the MVWT wireless testbed ( Jin et al. [2004]) at Caltech to demonstrate the results in the paper.

\section{REFERENCES}

Peter Alriksson and Anders Rantzer. Distributed kalman filtering using weighted averaging. Proc. 17th International Symposium on Mathematical Theory of Networks and Systems, Kyoto, Japan, 2006.

L. Bao, M. Skoglund, and K. H. Johansson. Encoderdecoder design for event-triggered feedback control over bandlimited channels. American Control Conference, Minneapolis, Minnesota, USA, 2006.

Dimitri P. Bertsekas and R. Gallager. Data Networks. Prentice Hall, 2nd edition, 1991.

A. Bonivento, C. Fischione, and A. SangiovanniVincentelli. Randomized protocol stack for ubiquitous networks in indoor environment. IEEE CCNC, 1, April 2006.

Vijay Gupta, Demetri Spanos, Babak Hassibi, and Richard M. Murray. On lqg control across a stochastic packet-dropping link. Proceedings of the American Control Conference, Portland, Oregon, 2005.

Joao Hespanha, Payam Naghshtabrizi, and Yonggang Xu. Networked control systems: Analysis and design. To appear in the Proc. of IEEE, Special Issue on Networked Control Systems, 2007.

Z. Jin, S. Waydo, E. B. Wildanger, M. Lammers, H. Scholze, P. Foley, D. Held, and Richard M. Murray. Mvwt-ii: The second generation caltech multi-vehicle wireless testbed. Proceedings of the American Control Conference, Boston, MA, 2004.

Wei Lai and Ioannis Ch. Paschalidis. Routing through noise and sleeping nodes in sensor networks: latency vs. energy trade-offs. pages $551-555$. Proceedings of the 45th IEEE Conference on Decision and Control, San Diego, CA, USA, Dec 2006.

Juan Liu, Feng Zhao, and Dragan Petrovic. Informationdirected routing in ad hoc sensor networks. IEEE Journal on Selected Areas in Communications, 23(4), April 2005.

R. Olfati-Saber. Distributed kalman filter with embedded consensus filters. Proceedings of the 44th IEEE Conference on Decision and Control and European Control Conference, 2005.

A. Panousopoulou, G. Nikolakopoulos, A. Tzes, and J. Lygeros. Experimental evaluation of a mobile adhoc networked (manet)controlled system. Proc. 17th International Symposium on Mathematical Theory of Networks and Systems, Kyoto, Japan, 2006.

B. S. Rao and H. F. Durrant-Whyte. Fully decentralised algorithm for multisensor kalman filtering. In IEE Proceedings-Control Theory and Applications, Sept 1991.

Ling Shi, Michael Epstein, Ahbishek Tiwari, and Richard.M.Murray. Estimation with information loss: Asymptotic analysis and error bounds. Proceedings of the IEEE Conference on Decision and Control and European Control Conference, 2005.

Ling Shi, Karl Henrik Johansson, and Richard M. Murray. Change sensor topology when needed: How to efficiently use system resources in control and estimation over wireless networks. Proceedings of the IEEE Conference on Decision and Control, New Orleans, 2007.

Drago Siljak. Large-Scale Dynamic Systems: Stability and Structure. North-Holland, New York, 1978.

B. Sinopoli, L. Schenato, M. Franceschetti, K. Poolla, M. Jordan, and S. Sastry. Kalman filtering with intermittent observations. IEEE Transactions on Automatic Control, 49(9):1453-1464, 2004.

D. Spanos, R. Olfati-Saber, and Richard M. Murray. Distributed kalman filtering in sensor networks with quantifiable performance. Proceedings of the 4th International Conference on Information Processing in Sensor Networks, 2005.

E. Witrant, P. G. Park, M. Johansson, C. Fischione, and K. H. Johansson. Predictive control over wireless multihop networks. Proceedings of the IEEE Conference on Control Applications, Singapore, 2007. 